\title{
Uso de una Estación Experimental de Lombricomposta para Desarrollar Experiencias Multidisciplinarias a Nivel Universitario
}

\author{
Mariana Ruiz ${ }^{(1)}$, Adriana Acevedo(2) \\ (1) Universidad Iberoamericana, Departamento de Ingenierías, Prolongación Paseo de la Reforma \\ 880, Lomas de Santa Fe, 01219, México D.F. -México (e-mail: mariana.ruiz@uia.mx) \\ (2) Universidad Iberoamericana, Departamento de Ingenierías, Prolongación Paseo de la Reforma \\ 880, Lomas de Santa Fe, 01219, México D.F. -México (e-mail: adriana.acevedo@uia.mx)
}

Recibido Abr. 26, 2011; Aceptado May. 17, 2011; Versión final recibida May. 30, 2011

\begin{abstract}
Resumen
Este trabajo describe el uso de una estación de lombricomposta en la Universidad Iberoamericana, Ciudad de México, como herramienta educativa para generar experiencias multidisciplinarias. En la estación han participado alumnos de cinco programas de licenciaturas y se han generado siete tipos de experiencias que han tenido como resultado el aumento en productividad de la estación de casi $500 \%$. Esta herramienta educativa ha permitido ilustrar temas de manejo de residuos, análisis químico, biología, impacto urbano, eficiencia y productividad entre otros y se espera que en el futuro cercano se sumen nuevas actividades en varios otros cursos para continuar promoviendo en el alumno el desarrollo de la práctica reflexiva, la preocupación por el medioambiente y el desarrollo tecnológico sustentable.
\end{abstract}

Palabras clave: lombricomposta, herramientas educativas, proyectos ecológicos, experiencias multidisciplinarias

\section{Using an Experimental Vermicomposting Station to Develop Multidisciplinary Experiences at College Level}

\begin{abstract}
This paper describes the use of a vermicomposting station at the Universidad Iberoamericana in Mexico City, as an educational tool to generate multidisciplinary experiences. Students from five majors have participated in the station in one or more of the seven experiments that have been designed for this purpose, and the station has increased its productivity in almost $500 \%$. This educational tool has allowed illustrating several topics on waste management, chemical analysis, biology, urban impact, efficiency and productivity, among others. In the near future, it is expected to incorporate new activities in several other courses, to continue promoting reflective practice, environmental concern, and sustainable technology development.
\end{abstract}

Keywords: vermicomposting, educational tools, ecological projects, multidisciplinary experiences 


\section{INTRODUCCIÓN}

Hoy en día existe una creciente preocupación por la cantidad de residuos que arrojamos al planeta. Como respuesta, las autoridades y diversas instituciones han empezado a considerar la práctica del composteo como una opción viable y eficaz para reducir significativamente la cantidad de residuos que se transportan a los vertederos. Las investigaciones sobre el aprovechamiento de los residuos municipales a través del composteo con lombriz datan desde finales de la década de 1970 principalmente en las universidades de Cornell y Syracuse, Estados Unidos, con estudios a cargo de Fong y Hewitt (2011). Algunos estudios sobre elaboración de humus de lombriz están disponibles en publicaciones de Bogdanov (1996), Ernst (1995), Nancarrow y Taylor (1998), y Wilson (1999). En México, la industria de la vermicultura se enfoca en sistemas agroindustriales a gran escala (composteo de residuos de café y bagazo de caña de azúcar, mezclas de biosólidos y estiércol), y existe una norma, la NMXFF-109-SCFI-2008 (SECOFI, 2008), que establece las especificaciones para el humus de lombriz (lombricomposta), incluyendo la designación y clasificación del producto, así como las especificaciones y los métodos de prueba necesarios para su comercialización.

El composteo in-situ ha aumentado su popularidad en los últimos años en diversas universidades en América del Norte (Simpson, 2008). Según información recopilada durante 2008 y 2009, diversas instituciones educativas afirman que los materiales orgánicos constituyen la mayor parte de sus residuos. En un estudio publicado por Fournier (2005) donde compila los logros en materia de reciclaje de diversas universidades de Estados Unidos resalta que un $75 \%$ de las universidades analizadas reciclan los residuos de jardinería y $45 \%$ los residuos de alimentos a través del composteo. Por otro lado, las universidades de Estados Unidos reportadas con proyectos de composteo específicamente con lombriz son University of Massachussets-Amherst, University of Oregon, University of Calgary, University of California - Davis, North Carolina State University y Colorado State University. Las universidades e institutos en México también están involucrados en el tema de lombricomposta. Diversas instituciones ofrecen cursos sobre elaboración de humus de lombriz -principalmente a través de programas de educación continua-, incluyendo la Universidad Autónoma de Chapingo, el Instituto Nacional de Ecología, la Universidad Nacional Autónoma de México y la Universidad Veracruzana. Al mismo tiempo, varias universidades tienen proyectos de composteo; entre las que se reportan en la literatura están la Universidad Autónoma Metropolitana, la Universidad Autónoma de Baja California y la Universidad de Sonora. Sin embargo, los únicos casos reportados de producción de lombricomposta a escala media son la Universidad de Sonora (2004) y la Universidad Iberoamericana (2011).

A pesar de la práctica extensiva del lombricomposteo en universidades, son pocas las publicaciones acerca del uso de este tipo de sistema como herramienta educacional a nivel licenciatura. Como ejemplo, Melear y Lunsford (2007) publicaron un artículo donde describen cómo una estación de lombrices usada de manera temática y a largo plazo, puede proporcionar experiencias profundas y significativas que, de manera simultánea, abarcan una gran variedad de temas científicos. Otro ejemplo lo presenta Kelley (2010) quien plantea cómo una cama de lombrices puede demostrar una diversidad de conceptos científicos y es una herramienta valiosa en clases de licenciatura. También se reportan en la literatura experiencias del uso de cajas de composteo dentro de los salones de educación primaria y secundaria principalmente en los Estados Unidos (Farrell, 1997; Trautmann, 1998). Aunque, según Melear y Lunsford (2007), se pueden obtener resultados similares con otro tipo de organismos tales como terrarios, acuarios, plantas o incluso cultivos bacterianos, un sistema de lombricomposta puede utilizarse para demostrar una variedad de temas mucho más amplia, incluyendo distintas ramas de la ingeniería y la promoción de actitudes y valores como la responsabilidad ambiental. Cabe señalar que ninguno de los trabajos anteriores reporta el uso de instrumentos para la evaluación cuantitativa de los resultados de aprendizaje derivados de estas experiencias.

Por otro lado, la promoción de la responsabilidad ambiental, también llamada educación en desarrollo sustentable, ha tomado gran impulso en las Universidades. Existe una publicación dedicada tan sólo a este tema: "The International Journal of Sustainability in Higher Education", y 
autores como Leal-Filho (2010) han hecho análisis extensos sobre las corrientes actuales y necesidades futuras en este tema de educación en desarrollo sustentable. Autores reconocidos han concluido que el tema de desarrollo sustentable debe ser integrado en los contenidos de los cursos de las ingenierías, más que añadido como un curso específico; pero al mismo tiempo han identificado dificultades entre los académicos para incluir este tema en sus propios cursos (Rydhagen y Dackman, 2011; Leal-Filho, 2010). Las principales barreras identificadas son que algunos académicos piensan que la sustentabilidad es todavía un tema abstracto y distante de la realidad e incluso demasiado amplio. Por ello, Leal-Filho (2010) propone que las Universidades aborden este tema a través de casos de estudio, proyectos piloto o iniciativas in-situ que demuestren lo cerca que está la sustentabilidad de la realidad. En particular, recomienda que en lugar de promover discusiones amplias y teóricas sobre la sustentabilidad, el alumno sea inmerso en temas específicos y de interés regional a fin de contextualizar y obtener resultados muy específicos de la sustentabilidad.

En el año 2007, un grupo de investigadores de la Universidad Iberoamericana, Ciudad de México, se interesó por la creación de un proyecto que permitiera a los alumnos analizar sistemas de índole ambiental desde una perspectiva interdisciplinaria y en 2008 se construyó la estación experimental de lombricomposta. A pesar de enfrentar una serie de retos para su funcionamiento, sobretodo en la fase inicial, en el año 2009 se alcanzó el estado estable del sistema con una población máxima de $80 \mathrm{~kg}$ de lombriz roja californiana (Eisenia fetida) y una producción de composta de $40 \mathrm{~kg}$ al mes en un área de $6.25 \mathrm{~m}^{2}$. La principal aportación del presente trabajo es que, aunque existen numerosos proyectos y estudios sobre lombricomposta, y numerosos estudios sobre la educación en desarrollo sustentable, no se encuentran en la literatura reportes que describan el uso del lombricomposteo como herramienta de educación en desarrollo sustentable que permita generar una serie de experiencias multidisciplinarias, y al mismo tiempo enfocadas y prácticas, a nivel licenciatura. El uso de estas experiencias de campo no es nuevo. Parte fundamental de muchos cursos sobre ciencias de la tierra y ciencias ambientales son los laboratorios y experiencias de campo, resaltados por Pasquier y Narguizian (2006), Connolly et al. (2006), entre otros. Sin embargo, la factibilidad de estas actividades puede verse limitada por cuestiones de distancia, tiempo, costo y seguridad. Ante esto, la estación experimental presenta una serie de ventajas: es accesible por encontrarse dentro del campus; es viable para su reproducción, pues no se requiere mucha inversión para su funcionamiento (los insumos provienen de la propia universidad); tiene un tamaño adecuado para poder ilustrar diversos conceptos (produce a mediana escala, no a micro escala, por lo que grupos grandes de alumnos pueden realizar diversos proyectos de impacto significativo simultáneamente) y, finalmente, tiene una vida larga que puede permitir al alumno tener contacto con ella a lo largo de varios semestres, reforzando el valor ambiental y transformándolo en un comportamiento duradero. Es decir que, además de la aplicación de conocimientos, pretendemos que a través de la práctica reflexiva del cuidado del ambiente realizada en esta estación, el alumno asimile verdaderamente dicho valor.

\section{METODOLOGÍA}

Utilizando el recurso existente de la estación experimental de lombricomposta, se diseñaron diversas actividades para promover el desarrollo de proyectos de corte interdisciplinario. Primero se seleccionaron algunas licenciaturas cuyos profesionistas suelen tomar decisiones de alto impacto en el tema de la sustentabilidad: ingenierías (por el tema de desarrollo de tecnología), arquitectura (en cuanto a urbanización) y diseño industrial (uso de diferentes materiales y recursos). A continuación, se buscaron aquellos cursos que, por su contenido, podrían incluir el tema de sustentabilidad sin forzarlo y, que a la vez, los conocimientos y técnicas pudieran dar resultados inmediatos en la estación de lombricomposta (cerrando el ciclo de retroalimentación inmediata para el alumno). Se identificaron 7 cursos distribuidos al inicio, en medio y al final de las licenciaturas y todos los alumnos inscritos en dichos cursos participaron de las experiencias diseñadas; es decir, no hubo una selección particular de los alumnos participantes (ver Tabla 1). El profesor-investigador encargado de la estación proporcionó a los alumnos los elementos teóricos y verificó la aplicabilidad de los proyectos. Los profesores titulares de cada uno de los cursos se encargaron de apoyar a los alumnos con conocimientos y herramientas propias del curso y orientar al alumno en su forma de aplicarlas en el proyecto. 
Tabla 1. Resumen de las experiencias, cursos y participantes (Periodo: enero 2009 - enero 2011).

\begin{tabular}{|l|l|l|l|l|}
\hline Experiencia & Curso & Licenciatura & Semestre & \# alumnos \\
\hline Visita y práctica de cosecha & $\begin{array}{l}\text { Introducción a la } \\
\text { Ingeniería }\end{array}$ & $\begin{array}{l}\text { Ingeniería } \\
\text { Industrial }\end{array}$ & 1er semestre & 140 alumnos \\
\hline $\begin{array}{l}\text { Prácticas para aumento de } \\
\text { productividad }\end{array}$ & $\begin{array}{l}\text { Estudio del Trabajo y } \\
\text { Productividad }\end{array}$ & $\begin{array}{l}\text { Ingeniería } \\
\text { Industrial }\end{array}$ & 3er semestre & 60 alumnos \\
\hline $\begin{array}{l}\text { Evaluación de impacto } \\
\text { ambiental }\end{array}$ & $\begin{array}{l}\text { Ingeniería Humana e } \\
\text { Impacto Ambiental }\end{array}$ & $\begin{array}{l}\text { Ingeniería } \\
\text { Industrial }\end{array}$ & $8^{\circ}$ semestre & 45 alumnos \\
\hline $\begin{array}{l}\text { Visita ilustrativa } \\
\text { Medio Natural y Entorno } \\
\text { Construido }\end{array}$ & Arquitectura & 1 er semestre & 80 alumnos \\
\hline $\begin{array}{l}\text { Concurso de diseño de } \\
\text { trituradora }\end{array}$ & Diseño Mecánico & $\begin{array}{l}\text { Ingeniería } \\
\text { Mecánica }\end{array}$ & $4^{\circ}$ semestre & 18 alumnos \\
\hline $\begin{array}{l}\text { Proyecto de diseño de } \\
\text { compostero }\end{array}$ & Diseño Industrial IV & $\begin{array}{l}\text { Diseño } \\
\text { Industrial }\end{array}$ & $6^{\circ}$ semestre & 5 alumnos \\
\hline $\begin{array}{l}\text { Análisis fisicoquímico del } \\
\text { producto }\end{array}$ & $\begin{array}{l}\text { Laboratorio de Ingeniería } \\
\text { Ambiental }\end{array}$ & $\begin{array}{l}\text { Ingeniería } \\
\text { Química }\end{array}$ & $8^{\circ}$ semestre & 10 alumnos \\
\hline
\end{tabular}

\section{RESULTADOS Y DISCUSIÓN}

A continuación se describen las actividades realizadas -que van desde la propia producción de composta, el análisis del impacto ambiental de los residuos, el diseño de equipo, las evaluaciones de control de calidad, hasta el análisis fisicoquímico del producto final- y las experiencias recogidas en la estación experimental en los años 2009 y 2010:

Visita y práctica de cosecha.

En esta visita, los estudiantes reciben una explicación global sobre el funcionamiento del sistema. Se les explica cómo ha ido evolucionando la estación de lombricomposta y cómo ha ido aumentando su productividad gracias a los conocimientos que han aplicado estudiantes de semestres más avanzados. De esta forma, los alumnos comprenden que lo que van a aprender en la carrera son herramientas que realmente pueden mejorar el desempeño de un sistema, cualquiera que éste sea. Finalmente, los estudiantes participan en la selección de residuos y la cosecha del producto final, como se muestra en la figura 1.

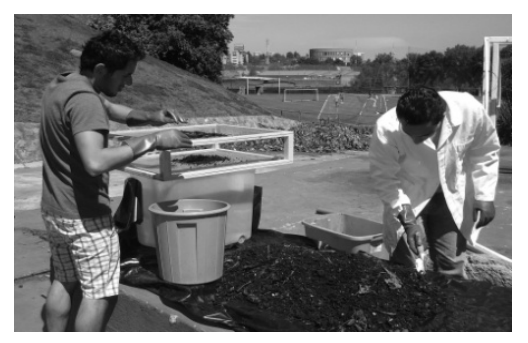

Fig. 1: Estudiantes participando en la cosecha de lombricomposta.

En los reportes redactados por los alumnos después de la visita a la estación, se encontraron comentarios reveladores sobre el impacto que tiene la experiencia de la estación en el alumno. Estos son algunos ejemplos:

"Pienso que el proceso de reciclaje por medio de lombricomposta puede ser un buen negocio a nivel industrial, ya que la materia prima son deshechos y el producto es abono, por lo que los insumos son muy baratos, mientras que el producto se puede vender a muy buen precio."

"La lombricomposta es algo que se debería aplicar en pequeña y gran escala, en los hogares así como en universidades y diferentes establecimientos. Con esto, crearíamos principalmente consciencia individual para poder dar el gran paso a la consciencia colectiva." 
"Los conocimientos que te da la Ingeniería Industrial son de gran utilidad para la generación de este proyecto. Temas tales como cadena de suministro y simulación se pueden aplicar en la lombricomposta, y más en caso de que se quiera realizar un modelo de negocio. Administrar tu inventario, distribución, ventas, etc., son cosas que se pueden resolver con las materias antes mencionadas."

"Apoyo enormemente este tipo de proyectos gracias a la filosofía que traen detrás, crear un mundo sustentable."

Prácticas para aumento de productividad de la estación.

En estas prácticas se aplican principios de la Ingeniería de Métodos para mejorar el desempeño de la estación. Para ello, los estudiantes hacen un análisis completo del sistema, elaborando diagramas de flujo, diagramas de recorridos y estudios de tiempos y movimientos en la estación. Una vez terminado el análisis, los alumnos aplican conceptos de reingeniería y proponen formas más económicas y eficientes de producir en la estación. A partir de estas prácticas, la estación ha aumentado su producción de composta en aproximadamente un 500\%, de $7.5 \mathrm{~kg}$ al mes a mediados de 2008, hasta los $40 \mathrm{~kg}$ al mes a finales de 2010. Este incremento en productividad nos ha demostrado que los alumnos han sido capaces de aplicar los conocimientos adquiridos en la materia a un proyecto real y con resultados satisfactorios. La aplicación de conocimientos ha sido significativa para los alumnos puesto que se construye la actividad sobre un tema ambiental, de gran atractivo entre los universitarios, coincidiendo con Garzón (1999) quien resalta la importancia de construir el conocimiento a partir de aquello que le interesa al alumno.

\section{Evaluación de impacto ambiental.}

Este curso incluye la evaluación del impacto ambiental tanto de las actividades cotidianas como las de los procesos industriales. Durante el curso, los alumnos se involucran en la cuantificación de materiales orgánicos generados en el campus universitario así como a nivel casero. Hacen un estudio del transporte de residuos hasta la estación experimental y presentan proyectos con propuestas para mejorar el proceso de manejo de materiales. Como resultado, se han colocado mejores señalizaciones para la captación de residuos reciclables en el campus y se ha podido capacitar de forma más eficiente a los encargados de la separación de residuos orgánicos para el composteo. Del grupo de la materia Ingeniería Humana e Impacto Ambiental impartida en el semestre de Primavera 2011, 18 alumnos fueron encuestados (5 mujeres y 13 hombres). A la indicación "Describe en un párrafo tu mayor aprendizaje de este curso", un $72 \%$ de los alumnos mencionó de forma positiva la experiencia con el proceso de composteo. De ellos, un 46\% manifestó que esta práctica los hizo más conscientes de las repercusiones de las actividades cotidianas y de lo que implica el reaprovechamiento de los recursos. Un 30\% hizo una mención directa del impacto en su compromiso y responsabilidad con el medio ambiente. El 15\% mencionó la palabra "sustentabilidad" como un objetivo alcanzable, y un $62 \%$ resaltó como mayor importancia el aspecto de aplicación práctica de la ingeniería. Es notable que el término "sustentabilidad" empieza a aparecer en las menciones libres del alumno, y no únicamente como concepto abstracto sino visto como un objetivo que puede ser alcanzado a través de acciones concretas. Las menciones sobre compromiso, responsabilidad y conciencia ambiental fueron notoriamente superadas por la mención de que el mayor aporte de la actividad es la aplicación práctica de la ingeniería.

\section{Visita ilustrativa.}

Los estudiantes en el curso Medio Natural y Entorno Construido deben considerar los retos que enfrentan las concentraciones urbanas y la generación, manejo y disposición de residuos sólidos. La actividad que se lleva a cabo con ellos incluye la visita a la estación experimental de lombricomposta durante la cual se les proporcionan estadísticas sobre generación de desechos y sus fuentes. A la vez, se les explica la técnica del lombricomposteo como opción para reducción de residuos y se les describe con detalle lo que otros alumnos de otras licenciaturas han realizado en la estación. Esto obedece a que autores como Leal-Filho (2009) han concluido que un fuerte obstáculo a la enseñanza del desarrollo sustentable es la falta de sinergia entre las ciencias, donde cada una se enseña como si no tuviera lazos con ninguna otra materia (la currícula no se ha hecho lo suficientemente trans-disciplinaria). Como resultado, tenemos que con esta visita se tienden lazos entre materias, y un $70 \%$ de los proyectos finales de los estudiantes que 
participaron en ella incluyen una propuesta interdisciplinaria de lombricomposteo como estrategia para lograr una ciudad sustentable.

Concurso de diseño de trituradora.

El objetivo de la materia Diseño Mecánico incluye la comprensión del funcionamiento de mecanismos básicos y en la aplicación de los principios de la ingeniería de materiales y de manufactura para el diseño de dispositivos. Durante 2009, los alumnos del curso se enfocaron al diseño de una máquina trituradora capaz de disminuir el tamaño de la partícula de los residuos vegetales a $10 \mathrm{~cm}$. o menos. La realización de prototipos como estos es una herramienta educacional muy efectiva para la adquisición y desarrollo de intuición y juicio profesional. Los prototipos permiten al alumno experimentar, obtener retroalimentación inmediata y tangible de sus esfuerzos, evaluar de manera crítica sus ideas de diseño y unir el conocimiento y teoría con la implementación práctica (Green, 2006). Como resultado de este curso, se diseñaron cuatro prototipos diferentes de máquinas trituradoras y, aunque en general las máquinas diseñadas molían en vez de triturar, el entusiasmo por el desarrollo de nuevos prototipos no decayó y se alcanzaron los objetivos de la materia al lograr que los alumnos diseñaran dispositivos viables, económicos, prácticos (por su tamaño y peso) y con los cálculos adecuados para dimensiones, formas y resistencia de materiales, las cuales se muestran en la figura 2.

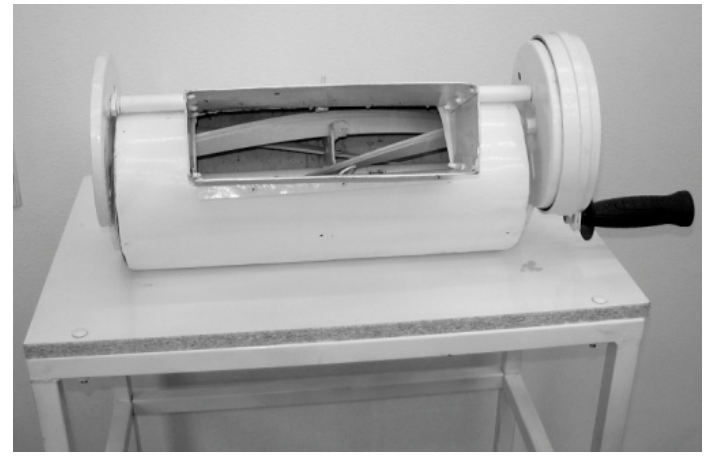

(a)

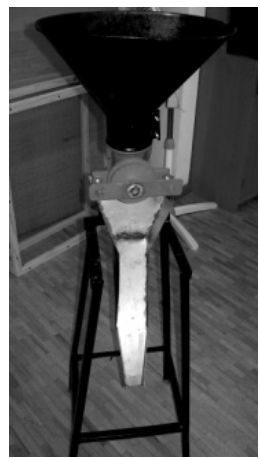

(b)

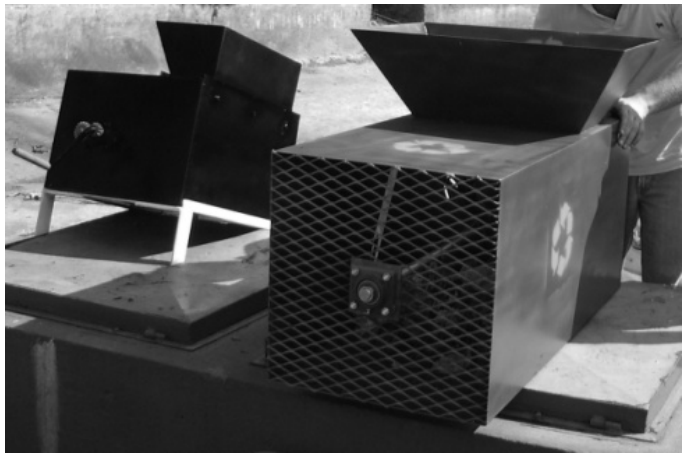

(c)

Fig. 2: Prototipos de máquinas trituradoras: a) a partir de una podadora de césped, b) a partir de un molino de maíz y c) dos trituradoras horizontales.

Proyecto de diseño de compostero.

Durante 2010, se solicitó a los alumnos de Diseño Industrial presentar por equipos una propuesta de un diseño original de un compostero vertical, incluyendo el modelo físico así como la descripción de materiales y costos. Como resultado, el proyecto elegido entre todos se encuentra actualmente construido y en periodo de prueba desde enero de 2011 . Durante el proceso, los alumnos pudieron aplicar sus habilidades en un proyecto totalmente real: hicieron investigación sobre el composteo, las lombrices y los materiales adecuados para ello, analizaron los materiales disponibles en el mercado, hicieron cálculos estructurales y de costos, logrando utilizar en un solo proyecto los conocimientos adquiridos en varias materias cursadas en su carrera. La figura 4 muestra uno de los diseños iniciales, y la figura 5 el prototipo construido.

Análisis fisicoquímico del producto.

Utilizando diversos métodos de prueba, los estudiantes llevan a cabo el análisis fisicoquímico de la composta terminada a fin de determinar si la muestra cumple con las normas mexicanas oficiales para este tipo de productos. Para este fin, los alumnos tienen que conocer a fondo la norma oficial y ser capaces de realizar análisis fisicoquímicos acordes con la misma. Terminado el análisis, los estudiantes escriben un reporte de laboratorio con todos los requerimientos y rigor de una publicación científica. Con dicho reporte se busca fomentar la competencia de comunicación científica en los alumnos. 


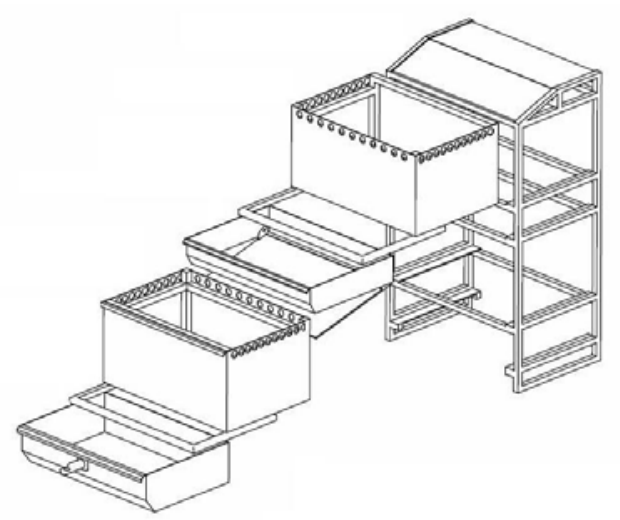

Fig. 4: Diseño de compostero vertical de dos módulos

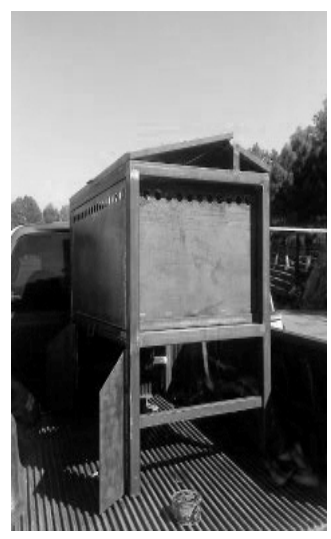

Fig. 5: Compostero vertical

En las 7 experiencias realizadas se observan resultados, ya sea de tipo cualitativo o cuantitativo, que muestran que el uso de la estación efectivamente promueve la aplicación práctica del conocimiento, el aprendizaje significativo y el reforzamiento de valores ambientales. Sin embargo, no se cuenta todavía con el instrumento de evaluación que sustente con validez y confiabilidad estadística dichas observaciones (coincidiendo con los autores que han realizado experiencias de aprendizaje similares como Melear y Lunsford, 2007 y Kelley, 2010, quienes no han utilizado ningún instrumento para medir la optimización del proceso de aprendizaje). Es por ello que el objetivo principal de la próxima etapa de este proyecto es desarrollar el instrumento de evaluación correspondiente, válido y confiable.

\section{CONCLUSIONES}

Gracias a las diferentes actividades realizadas en la estación de lombricomposta, los alumnos de distintos programas académicos a nivel licenciatura han aplicado conocimientos de áreas tan diversas como la química, la biología y la mecánica, hasta la economía y la planeación urbana. Al mismo tiempo, se ha tratado de reforzar en los alumnos los valores de la responsabilidad y compromiso con la naturaleza. Este reforzamiento de valores es de principal importancia en el quehacer de la Universidad Iberoamericana, Ciudad de México.

Primordialmente, son los alumnos de Ingeniería Industrial quienes tienen continuas experiencias con la estación en al menos tres materias a lo largo de su carrera. Esta repetición de la experiencia les puede permitir asimilar sus ventajas y retos desde distintas perspectivas por lo que se estima que esto generará un hábito de reducción de residuos. Sin embargo, es necesario cuantificar dicho impacto de manera objetiva, por lo que la próxima etapa en este proyecto consistirá en la generación de los instrumentos de evaluación que permitan medir de forma cuantitativa tanto la optimización del proceso enseñanza-aprendizaje como el reforzamiento del compromiso ambiental en el alumno.

En el futuro se espera sumar nuevas actividades en cursos diversos, a fin de continuar promoviendo no sólo la conciencia ambiental y la aplicación práctica de los conocimientos, sino el desarrollo de nuevas tecnologías con miras a la sustentabilidad.

\section{REFERENCIAS}

Bogdanov, P. Commercial vermiculture: How to build a thriving business in redworms. $1^{\text {st }}$ edition, 1-83, Vermico, Merlin, Oregon, USA (1996).

Connolly, R., Groome, M., Sheppard, K. y Stroud, N., Tips from the Field, Science Teacher, Vol. 73(1), 42-45 (2006). 
Ernst, D. The farmer's earthworm handbook: Managing your underground money-makers. $1^{\text {st }}$ edition, 1-112, Lessiter Publications, Brookfield, WI, USA (1995).

Farrell, Teaching children about vermicomposting, BioCycle, 38(6), 78-80 (1997).

Fong, J., y P. Hewitt. More about worms... and related classroom activities. Cornell Waste Management Institute, 2010. compost.css.cornell.edu/worms/moreworms.html. Acceso: 5 de abril (2011).

Fournier, M. Recycle This! Facilities Manager, Vol 1, January/February, 48-53 (2005).

Garzón, Carlos y Vivas, Mireya. Una didáctica constructivista en el aula universitaria. Educere, 3(5) , 1-11 (1999).

Green, G. y Smrcek, L., On the developing role of physical models in engineering design education, European Journal of Engineering Education, 31(2), 191-200 (2006).

Kelley, R. L. Worms in the college classroom: More than just a composting demonstration. Journal of College Science Teaching, 39(3), 52-55 (2010)

Leal-Filho, W., Education for sustainable development: current discourses and practices and their relevance to technology education, International Journal of Technology \& Design Education, 19(2), 149-165 (2009).

Leal-Fillho, W., Teaching sustainable development at university level: current trends and future needs, Journal of Baltic Science Education, 9(4), 273-284 (2010).

Melear, C.T. y Lunsford, E. Worms cultivate our curriculum: A long-term, theme-based Unit. Science Activities, 44(2), 48-54 (2007).

Nancarrow, L. y Taylor, J.H. The worm book. $1^{\text {st }}$ edition, 1-150, Ten Speed Press, Berkeley, California, USA (1998).

Pasquier y Narguizian, Using Nature as a Resource; Effectively Planning an Outdoor Field Trip. Science Activities, 43(2), 29-33 (2006).

Rydhagen, B. y Cackman, C., Integration of sustainable development in sanitary engineering education in Sweden, European Journal of Engineering Education, 36(1), 87-95 (2011).

SECOFI. Norma Mexicana. NMXFF-109-SCFI-2008 Humus de Lombriz (Lombricomposta)Especificaciones y Métodos de Prueba. Secretaría de Comercio y Fomento Industrial. Diario Oficial de la Federación, 26 de mayo (2008).

Simpson, W. The Green Campus: Meeting the Challenge of Environmental Sustainability. 1a ed., 1-361, Appa Assn of Higher Education, Alexandria, VA, USA, (2008).

Trautmann, N.M. y Krasny, M.E. Composting in the Classroom. 1a ed. 1-115, Kendall/Hunt, Dubuque, lowa, USA (1998).

Universidad de Sonora. Noticias: Experimenta Unison recuperación de suelos con lombricomposta, 11 de agosto de 2004, http://www.uson.mx/noticias/default.php?id=944. Acceso: 11 de noviembre (2009).

Universidad Iberoamericana. Boletín electrónico de Investigación de la Universidad Iberoamericana, Ciudad de México, 28 de febrero de 2011, http://www.uia.mx/web/files/inv/Investigacion_UIA_26.pdf. Acceso: 14 de marzo (2011).

Wilson, E. Worm farm management: Practices, principles, procedures. $1^{\text {st }}$ edition, 1-120, Kangaroo Press, Sydney, Australia (1999). 